Check for updates

Cite this: RSC Adv., 2018, 8, 5493

\title{
Self-assembled organic nanorods for dual chemo-photodynamic therapies $\uparrow$
}

\author{
Yuanyuan Li, ${ }^{a}$ Xiuli Hu, DD ${ }^{* b}$ Xiaohua Zheng, ${ }^{c}$ Yang Liu, ${ }^{d}$ Shi Liu, ${ }^{b}$ Ying Yue*a \\ and Zhigang Xie (iD) ${ }^{\mathrm{b}}$
}

Photodynamic therapy (PDT) and chemotherapy have been extensively developed as effective approaches against cancer. Herein, we constructed organic nanorods by rational co-assembly of photosensitizer, di-iodinated borondipyrromethene $\left(B D P-I_{2}\right)$, and chemical anticancer drug, paclitaxel (PTX). The physico- and photochemical properties of the obtained nanorods were carefully investigated. BDP- $I_{2}$ was selected for its high singlet oxygen $\left({ }^{1} \mathrm{O}_{2}\right)$ quantum yields. And the corresponding ${ }^{1} \mathrm{O}_{2}$ generation ability and photodynamic effect were evaluated both in vitro and in vivo. The accelerated endosomal escape of the nanorods induced by the photodynamic effect enhanced the chemotherapeutic efficacy of PTX. We believe that this synergetic nanomedicine represents a new development for antitumor chemophotodynamic therapy.

Received 4th January 2018

Accepted 25th January 2018

DOI: $10.1039 / \mathrm{c} 8 \mathrm{ra} 00067 \mathrm{k}$

rsc.li/rsc-advances

loading contents and ratios are uncontrollable. Furthermore, the carrier-related side toxicities also remain concerning. ${ }^{14}$ It is high challenging to develop nanoplatforms that combine chemotherapy and PDT with controlled drug ratios and limited excipients.

Borondipyrromethene (BODIPY) and its derivatives have received much attention and been considered as potential candidates as both bioimaging and PDT therapeutic agents owing to their excellent photostability, high molar extinction coefficient and quantum yields. ${ }^{14-20}$ Halogen atoms are commonly introduced for their efficient triplet-sensitization to BODIPY. ${ }^{15}$

Direct assembly of organic molecules has been proved to be a facile and useful method to obtain nanoplatforms with high drug loading and precise specific molecular structure. ${ }^{21-25}$ Morphology plays an important role in the performance of nanomaterials. Nanoparticles with different size and surface charge have been widely investigated. In our previous study, rod-shaped particles appear to be more favorably engulfed by cells compared to spherical counterparts. ${ }^{16}$ Herein, we construct nanorods (Co-NRs) by the co-assembly of BDP- $\mathrm{I}_{2}$ and paclitaxel (PTX) as shown in Scheme 1. The synthesis, characterization, and cellular behavior of the obtained Co-NRs are investigated. And the in vivo and in vitro photodynamic and chemotherapy effects are also evaluated.

\footnotetext{
${ }^{a}$ The First Hospital of Jilin University, Xinmin Street, Changchun, Jilin 130021, PR China.E-mail: yying119@126.com

${ }^{b}$ State Key Laboratory of Polymer Physics and Chemistry, Changchun Institute of Applied Chemistry, Chinese Academy of Sciences, Changchun 130022, China. E-mail: lily@ciac.ac.cn

${ }^{c}$ University of Science and Technology of China, Hefei 230026, PR China

${ }^{d}$ Department of Chemistry, Northeast Normal University, 5268 Renmin Street, Changchun 130024, P. R. China

$\dagger$ Electronic supplementary information (ESI) available. See DOI: 10.1039/c8ra00067k
}

\section{Results and discussion}

BDP- $\mathrm{I}_{2}$ was synthesized according to the previously reported method. ${ }^{26}$ The ${ }^{1} \mathrm{H}$ NMR spectroscopy and MALDI-TOF mass spectrum confirmed its accurate chemical structure (Fig. S1, ESI $†$ ). BDP-I $\mathrm{I}_{2}$ could self-assemble or co-assemble with 
A

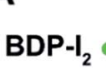

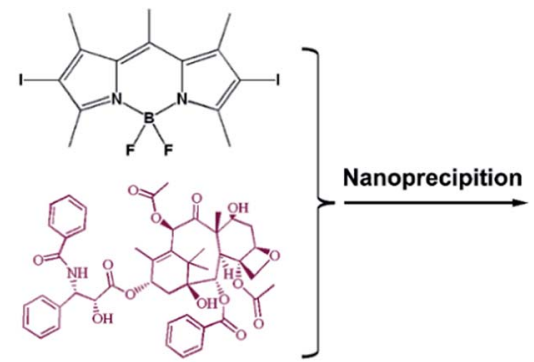

B

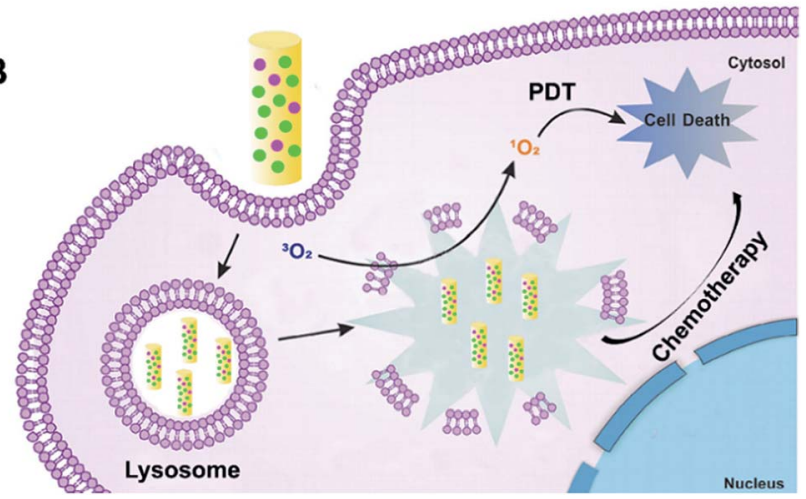

Scheme 1 Schematic illustration of the light-activated Co-NRs. (A) Schematic design of Co-NRs. (B) Photodynamic therapy of Co-NRs.

PTX into nanorods (NRs). The concentration of BDP- $\mathrm{I}_{2}$ and PTX in Co-NRs were determined to be $10 \mu \mathrm{g} \mathrm{mL} L^{-1}$ and $2 \mu \mathrm{g} \mathrm{mL} L^{-1}$, respectively, by UV-vis standard curve and high performance liquid chromatography (HPLC) (Fig. S2, ESI $\dagger$ ). Transmission electron microscopy (TEM) images confirmed that both BDP-I ${ }_{2}$ NRs and Co-NRs possessed rod morphology with a width of less than $200 \mathrm{~nm}$. Meanwhile, dynamic light scattering (DLS) profiles identified that the corresponding hydrodynamic diameters of BDP-I $\mathrm{I}_{2}$ NRs and Co-NRs were 195.8 and $211.5 \mathrm{~nm}$,
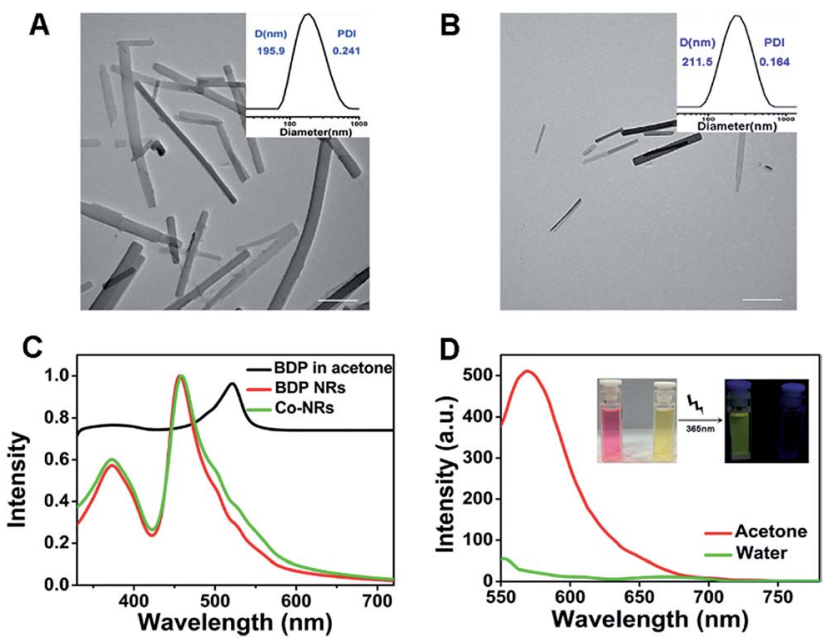

Fig. 1 The characterization of BDP-I $I_{2}$ NRs and Co-NRs. TEM images and DLS results of (A) BDP- $I_{2}$ NRs and (B) Co-NRs. Scale bars, $500 \mathrm{~nm}$. (C) Normalized UV-vis absorption of BDP- $I_{2}$ in acetone, BDP- $I_{2} \mathrm{NRs}$ and $\mathrm{Co}-\mathrm{NRs}$ in water. (D) Fluorescence spectra of BDP-I $\mathrm{I}_{2}$ in acetone and $\mathrm{BDP}-\mathrm{I}_{2} \mathrm{NRs}$ in water. Insets: photographs under UV light. respectively (Fig. 1A-B). Moreover, the size and size distribution of Co-NRs in water almost kept unchanged up to two weeks at room temperature, and kept unchanged for 7 days in 10\% PBScontaining water solution or $10 \%$ fetal bovine serum (FBS) containing water solution, indicating the good stability of asprepared Co-NRs in physiological conditions (Fig. S3A-C, ESI $\dagger$ ). The zeta potential of Co-NRs also showed not obvious fluctuation after two weeks (Fig. S3D, ESI†). All these results demonstrated the favorable physiological properties of Co-NRs.

Following, the photophysical properties of Co-NRs and BDP$\mathrm{I}_{2}$ were tested. UV-vis absorption spectra revealed that the maximum absorption peak of BDP-I ${ }_{2}$ changed from $521 \mathrm{~nm}$ in acetone to $459 \mathrm{~nm}$ in NR state (Fig. 1C) due to its aggregation into nano-rods. The Co-NRs and BDP-I $\mathrm{I}_{2}$ NRs showed almost the same UV-vis absorbance, indicating that adding PTX did not influence the absorbance of BDP-I $\mathrm{I}_{2}$ NRs. The fluorescence peak of BDP- $\mathrm{I}_{2}$ small molecule in acetone was $569 \mathrm{~nm}$ excited at $521 \mathrm{~nm}$. However, Co-NRs exhibited almost no fluorescence due to the aggregation-induced quenching (Fig. 1D). The inserted mage in Fig. 1D exhibited the photographs of the florescence difference excited at $365 \mathrm{~nm}$.

Indocyanine Green (ICG) and $2^{\prime}, 7^{\prime}$-dichlorodihydrofluorescein diacetate (DCFH-DA) were used as the indicators to investigate the reactive oxygen species (ROS) generation ability monitored by UV-vis absorption spectra. As shown in Fig. 2A-B, the maximum absorption of ICG at $778 \mathrm{~nm}$ showed a timedependent decrease upon irradiation due to oxidation of ICG by ROS produced by BDP-I $\mathrm{I}_{2}$. In contrast, ICG alone was stable under light irradiation, indicating the generation of ROS by BDP- $\mathrm{I}_{2}$ in Co-NRs. As the photostability of photosensitizers (PSs) was very important for the long time application in biology, ${ }^{27-30}$ the photostability of BDP-I $\mathrm{I}_{2}$ in Co-NRs was also tested. As shown in Fig. S4 (ESI $\dagger$ ), the absorption of Co-NRs almost kept unchanged under irradiation with a $540 \mathrm{~nm}$ lamp at $13 \mathrm{~mW} \mathrm{~cm}^{-2}$ for $20 \mathrm{~min}$, indicating its excellent photostability.
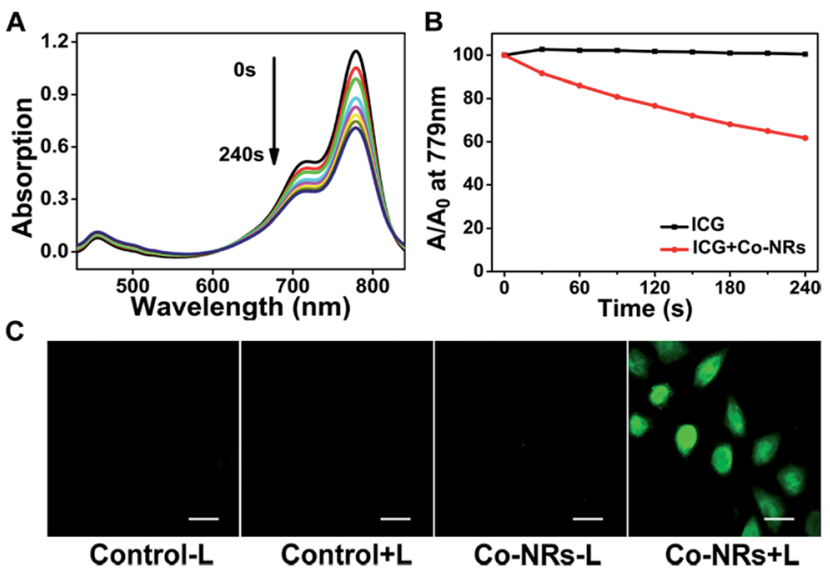

Fig. 2 The ROS generation ability of the Co-NRs. (A) Time-dependent UV-vis absorption spectra of ICG at $779 \mathrm{~nm}$ upon irradiation with a $540 \mathrm{~nm}$ LED light from 0 to $240 \mathrm{~s}$. (B) Comparison of decay rates of ICG alone and blending of ICG and Co-NRs upon irradiation. (C) Generation of ROS in vitro upon irradiation $\left(540 \mathrm{~nm}, 13 \mathrm{~mW} \mathrm{~cm}{ }^{-2}\right.$, $20 \mathrm{~min}$ ) indicated by the fluorescence of DCF. Scale bars, $20 \mu \mathrm{m}$. 
Besides, the intracellular ROS generation by Co-NRs was further tested in cervical cancer cells (HeLa cells) by using confocal laser scanning microscopy (CLSM). As shown in Fig. 2C, almost no green fluorescence appeared in the three control groups, indicating there was no adequate ROS generation in cells to oxidize no-fluorescent DCFH-DA to fluorescent dichlorofluorescein (DCF). ${ }^{31-38}$ In contrast, bright green fluorescence emerged in Co-NRs group with light irradiation, suggesting the generation of ROS.

The cellular uptake of Co-NRs by HeLa cells was studied by CLSM. The cellular nuclei were stained with DAPI to blue. With the time prolonged from $0.5 \mathrm{~h}$ to $6 \mathrm{~h}$, enhanced fluorescence was observed (Fig. S5, ESI $\dagger$ ). The fluorescence of Co-NRs decreased obviously when the incubation temperature changed from $37{ }^{\circ} \mathrm{C}$ to $4{ }^{\circ} \mathrm{C}$, indicating the ATP-dependent endocytosis pathway ${ }^{39-42}$ (Fig. S5, ESI $\dagger$ ). With light irradiation, a brighter fluorescence appeared for Co-NRs than the control group, suggesting the enhanced cellular uptake due to the photochemical internalization (PCI) effect..$^{13,43-48}$ (Fig. S5, ESI $\dagger$ ).

Lyso-tracker Red was employed as a probe to confirm the lysosome colocalization and escape of Co-NRs from lysosome. ${ }^{49-56}$ As shown in Fig. 3A, after incubation with Co-NRs for $2 \mathrm{~h}$, HeLa cells were stained with Lyso-tracker Red at $37^{\circ} \mathrm{C}$ for $30 \mathrm{~min}$. The red fluorescence co-localized well with the green fluorescence. After irradiation, enhanced green fluorescence and decreased red fluorescence were observed, confirming that the light irradiation facilitated the escape of Co-NRs from lysosome to cytoplasm due to the photochemical rupture of lysosome membranes (Fig. 3A-B). Lysosome escape further guaranteed the chemotherapeutic effect of PTX in Co-NRs.

Additionally, Acridine Orange (AO) staining test further confirmed the lysosome escape. ${ }^{57-60}$ In lysosomes, the concentrated AO emitted a granular red fluorescence, and a diffuse green fluorescence in cytosol. With the decreased red fluorescence and the increased green fluorescence upon light

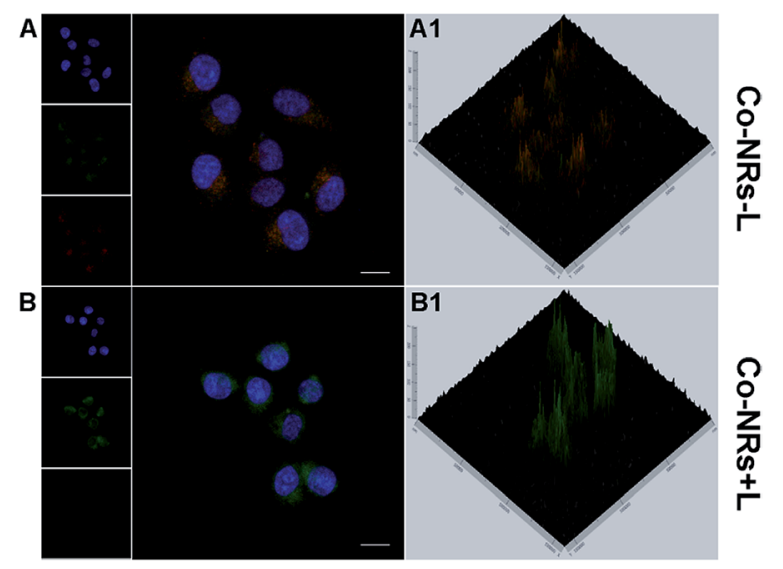

Fig. 3 CLSM images of HeLa cells incubated with Co-NRs in the presence of Lyso-tracker Red without $(-\mathrm{L})$ or with $(+\mathrm{L})$ light irradiation. (A1-B1) Fluorescence of Co-NRs and Lyso-tracker Red in cells at 2.5D mode. For each panel, the images from above to down show cell nuclei stained by DAPI (blue), fluorescence of Co-NRs (green), lysosomes stained with Lyso-tracker Red (red) and overlays of three images. Scale bars, $20 \mu \mathrm{m}$. irradiation, the relocation of $\mathrm{AO}$ from the lysosomes to the cytosol occurred, indicating the change of lysosome permeability due to the generated ROS (Fig. S6, ESI $\dagger$ ).

The in vitro cytotoxicity of Co-NRs against HepG2 and HeLa cells were evaluated by standard thiazolyblue tetrazolium bromide (MTT) assay. As shown in Fig. 4A, BDP-I ${ }_{2}$ NRs exhibited a negligible cytotoxicity without irradiation at the concentration of up to $1 \mu \mathrm{g} \mathrm{mL} \mathrm{m}^{-1}$ after $48 \mathrm{~h}$ incubation, implying the favorable biocompatibility of BDP-I ${ }_{2}$ NRs and excluding its dark cytotoxicity. Under irradiation with a lamp of $540 \mathrm{~nm}$, a dosedependent cytotoxicity of Co-NRs was observed against both HepG2 and HeLa cells. Co-NPs without irradiation can lead to cell death because of the antitumor effect of PTX. Moreover, under irradiation, Co-NRs exhibited a higher cytotoxicity than that of free PTX (Fig. 4B). The corresponding half-maximal inhibitory concentrations $\left(\mathrm{IC}_{50}\right)$ of Co-NRs without light, free PTX and Co-NRs plus light irradiation against HeLa cells were $0.06,0.013$ and $0.012 \mu \mathrm{g} \mathrm{mL}{ }^{-1}$, respectively. The similar cytotoxicity was also observed against HepG2 cells (Fig. 4C) and the corresponding $\mathrm{IC}_{50}$ values of Co-NRs without light, free PTX and Co-NRs plus light irradiation against HepG2 cells were listed in Table S1 (ESI $\dagger$ ). Furthermore, the anticancer efficiency of Co-NRs was also examined by the live/dead cell staining test (Fig. 4D). In the control groups, light irradiation alone and BDP$\mathrm{I}_{2}$ NRs in dark almost did not induce cell death. However, light irradiation could enhance the therapeutic effect of Co-NRs via photosensitizer-mediated lysosome escape. These results were in accordance with those of MTT.

The therapeutic effect of Co-NRs was further evaluated in vivo. Human cervical tumor xenograft (HeLa) in nude mice was utilized as the animal model. The obtained tumor-bearing mice were randomly divided into three groups: PBS plus light irradiation, Co-NRs without light irradiation and Co-NRs plus light irradiation. After intra-tumor injection of Co-NRs at the doses of $1.9 \mathrm{mg} \mathrm{kg}^{-1}$, a $450 \mathrm{~nm}$ lamp at $200 \mathrm{~mW} \mathrm{~cm}^{-2}$ was employed to irradiate the tumors for $10 \mathrm{~min}$, followed by measuring the tumor volume and body weight. As shown in Fig. 5A-F, PBS plus light irradiation group displayed a sharp tumor growth. In the absence of irradiation, Co-NRs had a relatively satisfied tumor inhibition effect as compared to that of control group due to the favorable therapeutic effect of PTX (Fig. 5B). With the treatment of irradiation, tumors of Co-NRs group were almost completely inhibited (Fig. 5C). After 20 days of observation and measurements, the tumors were harvested from the three groups. Statistical analysis demonstrated that the weights and sizes of tumors treated with Co-NRs were significantly smaller than the control group (Fig. 5E and F). Moreover, as shown in Fig. 5G, the body weights of mice had no significant difference after various treatments, indicating the low side effects and noticeable system toxicity of Co-NRs.

\section{Experimental section}

\section{Materials and characterization}

Milli-Q water was obtained from a Milli-Q system (Millipore, USA). Chemicals and reagents were acquired from commercial sources without further purification. Indocyanine green (ICG) 
A

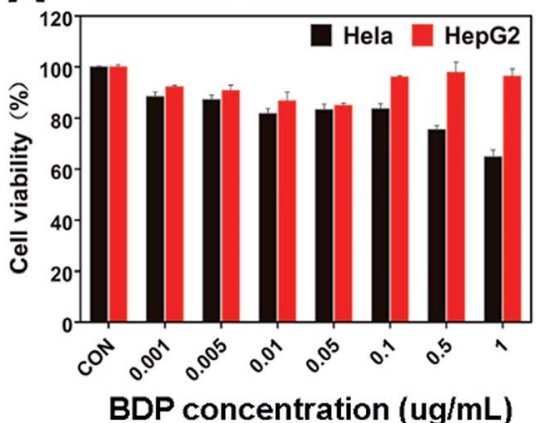

B

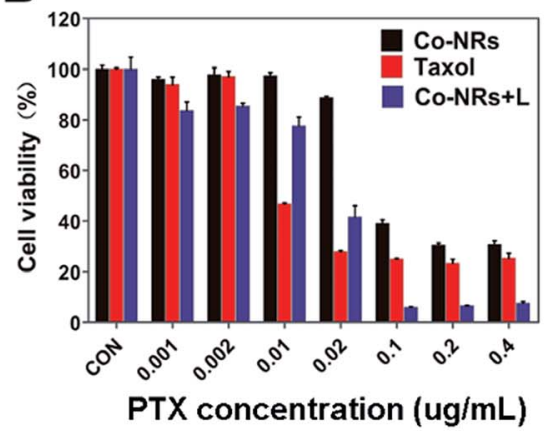

C

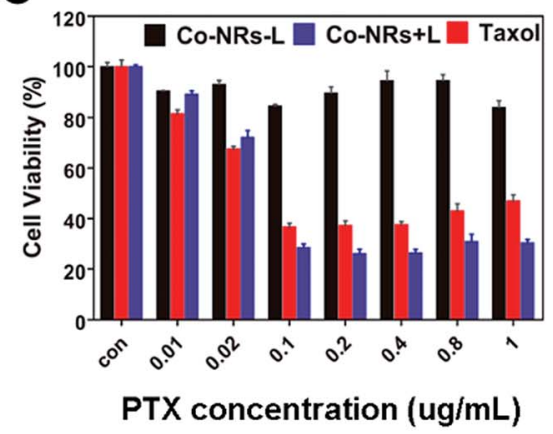

D Control + L BDP- $\mathrm{I}_{2} \mathrm{NRs}$ - L Co-NRs - L BDP-I 2 NRs + L Co-NRs + L
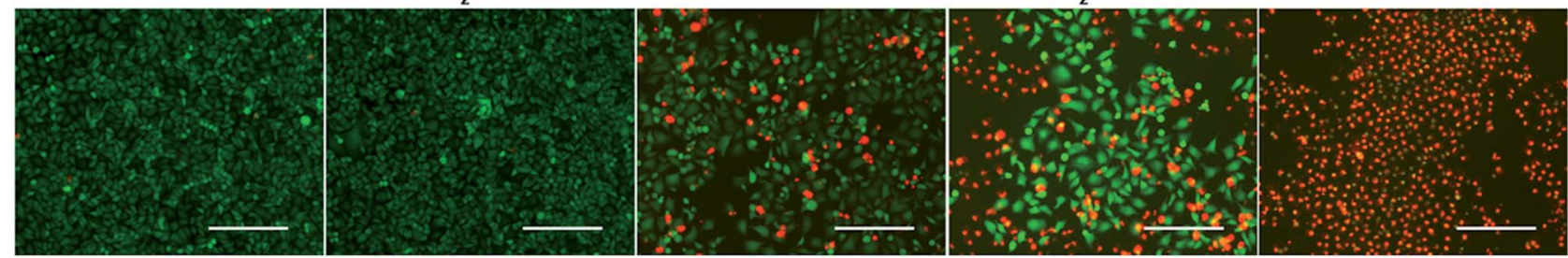

Fig. 4 In vitro synergistic therapy efficacy of Co-NRs. (A) Relative cell viabilities of HepG2 and HeLa cells incubated with BDP-I 2 NRs. Cell viabilities of HeLa (B) and HepG2 cells (C) incubated with Co-NPs upon a $540 \mathrm{~nm}$ lamp irradiation at $13 \mathrm{~W} \mathrm{~cm} \mathrm{~cm}^{-2}$ for 10 min. (D) Fluorescence microscope images of calcein-AM (green, live cells) and propidium iodide (red, dead cells) co-cultured HeLa cells pretreated with various conditions. Scale bars, $40 \mu \mathrm{m}$.

A

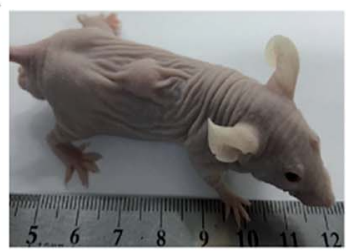

E

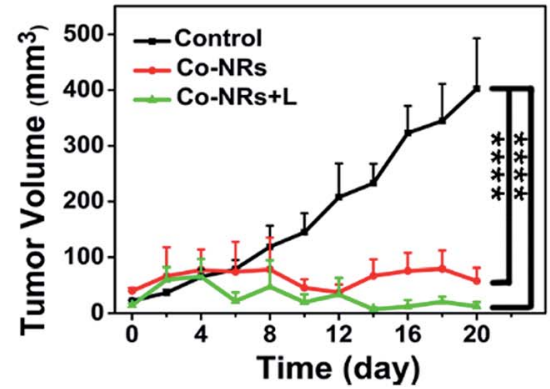

C

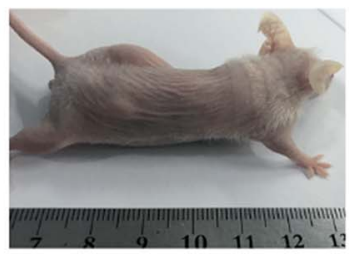

$\mathbf{F}$

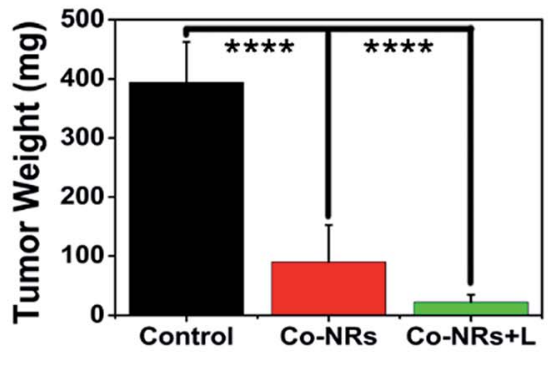

D

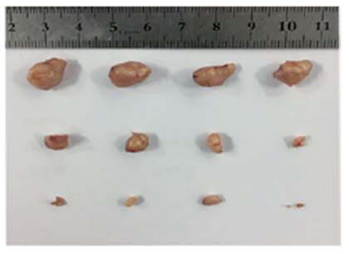

G

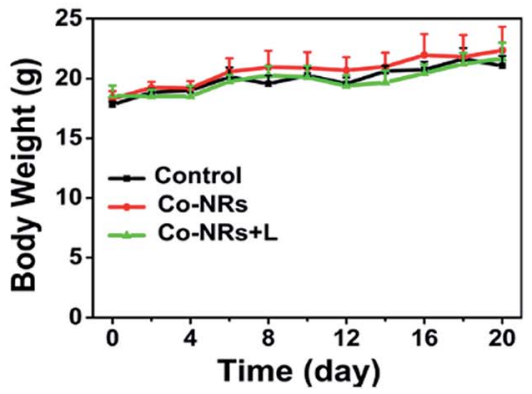

Fig. 5 In vivo anticancer efficacy. Various groups treated with (A) PBS + light, (B) Co-NRs in dark, (C) Co-NRs + light. (D) Photo of excised tumors. From top to down: PBS + light, Co-NRs in dark and Co-NRs + light group. (E) Changes of tumor volumes of three groups. (F) Tumor weights of three groups. (G) Body weights of mice after different treatments. Statistical significance: $* * * * P<0.0001$.

was purchased from Fisher Scientific. Dulbecco's Modified Eagle Medium (DMEM), RPMI-1640 Medium, and fetal bovine serum (FBS) were purchased from Sigma-Aldrich. LysoTracker Red and DAPI were purchased from Life Technologies. LiveDead Cell Staining Kit was purchased from KeyGen Biotech Co., Ltd. ${ }^{1} \mathrm{H}$ NMR spectra were recorded on an Agilent Mercury $400 \mathrm{MHz}$ spectrometer in chloroform-d. Transmission electron microscopy (TEM) images were taken by a JEOL JEM-1011
(Japan) at the accelerating voltage of $100 \mathrm{kV}$. The fluorescence emission spectra were obtained from a Hitachi fluorometer (F-7500 model). The UV-vis spectroscopy study was performed on a Shimadzu UV-vis spectro-photometer (UV-1800 model). Confocal microscopy imaging was performed using a Zeiss LSM 700 confocal and images were analyzed using ImageJ (NIH). 


\section{Preparation of BDP-I ${ }_{2}$ and paclitaxel (PTX) co-assembly} nanorods (Co-NRs)

Co-NRs were prepared by the reprecipitation method. Firstly, the mixture of BDP- $\mathrm{I}_{2}$ and PTX were dissolved in acetone $(4 \mathrm{~mL})$, then milli-Q water was added dropwise into the solution under stirring. After an overnight stirring, the Co-NRs were obtained and characterized. The BDP-NRs were obtained using the similar method without adding PTX.

\section{Cell culture}

HeLa cells (the human cervical cancer cell line) and HepG2 cells (the human liver hepatocellular carcinoma cell line) were purchased from Jilin University and cultured in culture medium containing DMEM (Dulbecco's modified Eagle's medium), 10\% FBS (fetal bovine serum), $100 \mathrm{U} \mathrm{mL}^{-1}$ penicillin, and $100 \mathrm{U} \mathrm{mL}^{-1}$ streptomycin. The temperature was $37^{\circ} \mathrm{C}$. The concentration of $\mathrm{CO}_{2}$ was $5 \%$.

\section{Biocompatibility of BDP and BDP-NRs in vitro by MTT assay}

HeLa cells and HepG2 cells were harvested in a logarithmic growth phase and then seeded in 96-well plates at a density of $2 \times 10^{5}$ cells per well. After incubation for $24 \mathrm{~h}$, the medium was replaced with medium containing with drugs, $200 \mu \mathrm{L}$ per well. Each concentration was set 4 repetitions. Then the light group received irradiation for $10 \mathrm{~min}$, the dark control group received nothing. The green lamp with a wavelength of $540 \mathrm{~nm}$ at an intensity of $13 \mathrm{~mW} \mathrm{~cm}^{-2}$ was used to irradiate. After 48 hours, MTT assay was used to measure the cell viability, using the microplate reader and reading at $490 \mathrm{~nm}$.

\section{Endolysosome escaping and AO staining}

HeLa cells were seeded in 6-well plates with cover slips. After incubation for $24 \mathrm{~h}$, the origin medium was replaced by drugcontaining medium (BDP-I $\mathrm{I}_{2}$ at $0.1 \mu \mathrm{g} \mathrm{mL}{ }^{-1}$ ), 2 hours later, the light group received irradiation for $20 \mathrm{~min}$. Lyso-tracker Red diluted in PBS was added, and the cells were further incubated at $37{ }^{\circ} \mathrm{C}$ for $45 \mathrm{~min}$. Then the samples were observed with CLSM.

As for AO staining, the plates without slips were used. After the incubation with drug (BDP- $\mathrm{I}_{2}$ at $0.1 \mu \mathrm{g} \mathrm{mL}{ }^{-1}$ ), light group was treated with irradiation for $20 \mathrm{~min}$. The cells were washed with PBS gently, and then stained with acridine orange (AO, $2.5 \mathrm{mg} \mathrm{mL}{ }^{-1}$ ), and incubated for another $15 \mathrm{~min}$ at $37{ }^{\circ} \mathrm{C}$. Fluorescence microscope was employed to observe the images.

\section{ROS detection}

CLSM was used to investigate the generation of intracellular ROS. HeLa cells obtained from the logarithmic growth phase were seeded in 6-well plate with cleaned cover slip. After an incubation of $24 \mathrm{~h}$, we switched the culture medium with medium with drugs or not. The final BDP- $\mathrm{I}_{2}$ concentration was $0.1 \mu \mathrm{g} \mathrm{mL}^{-1}$. Two hours later, the light group received an irradiation of $20 \mathrm{~min}$. The cells were washed immediately with DMEM solution without FBS, and then, the DMEM solution containing DCFH-DA was added as a sensor. The cover slips were observed with CLSM as soon as possible after further incubation for $45 \mathrm{~min}$ at $37^{\circ} \mathrm{C}$. The whole process was carried out dark in case of the quenching. The excitation wavelength of CLSM was $488 \mathrm{~nm}$, and the emission band pass was 500-550 nm.

Furthermore, ICG and UV-vis spectroscopy were employed to evaluate the singlet oxygen generation ability in vitro. ICG water solution $\left(1 \mathrm{mg} \mathrm{mL}^{-1}\right)$ was prepared at first. Then $21 \mu \mathrm{L}$ of the solution was added into $3 \mathrm{~mL}$ of water and $3 \mathrm{~mL}$ of Co-NRs (BDP-I ${ }_{2}$ concentration at $5 \mu \mathrm{g} \mathrm{mL}^{-1}$ ), separately. Both samples received an irradiation of $240 \mathrm{~s}, 30 \mathrm{~s}$ per time, and were detected 9 times. The changes in $779 \mathrm{~nm}$ were analyzed.

\section{Animal study}

Male nude mice were purchased from Jilin University, China (56-84 d, 15-20 g) and maintained under control. All the experiments were performed in strict accordance with the NIH guidelines for the care and use of laboratory animals (NIH Publication No. 85-23 Rev. 1985) and was approved by the guidelines of the Committee on Animal Use and Care of Chinese Academy of Sciences. Hela cells $(100 \mu \mathrm{L})$ were injected into the back of nude mice. One week later, $200 \mu \mathrm{L}$ of drugs were intratumoral injected into the tumors. The control group received PBS instead. Two hours later, the control and light group received an irradiation of $450 \mathrm{~nm}, 200 \mathrm{~mW} \mathrm{~cm}{ }^{-2}$, for $10 \mathrm{~min}$. In the next 20 days, body weight and tumor volume of mice were measured every two days. Finally, the tumors were harvested and weighed.

\section{Conclusions}

In summary, a nanotheranostic agent was successfully prepared by the co-assembling of photosensitizer BDP-I ${ }_{2}$ and chemical anticancer drug PTX. The obtained Co-NRs could effectively be internalized by cells and enhance the lysosome escape of PTX, promote the cell cytotoxicity in vitro. In vivo experiments further validated the excellent therapeutic efficacy of as-prepared CoNRs. This present work emphasizes the progress in the field of small molecule self-assembly and helps to elevate these to later clinical trials.

\section{Conflicts of interest}

There are no conflicts to declare.

\section{Acknowledgements}

This work was supported by the National Natural Science Foundation of China (Project No. 51773197 and 51522307).

\section{Notes and references}

1 N. Goswami, Z. Luo, X. Yuan, D. T. Leong and J. Xie, Mater. Horiz., 2017, 4, 817-831.

2 X. Li, Z. Yang, N. Hu, L. Zhang, Y. Zhang and L. Yin, RSC Adv., 2016, 6, 48379-48386. 
3 Z. Zhou, J. Song, L. Nie and X. Chen, Chem. Soc. Rev, 2016, 45, 6597-6626.

4 S. S. Lucky, K. C. Soo and Y. Zhang, Chem. Rev., 2015, 115, 1990-2042.

5 C. Qian, J. Yu, Y. Chen, Q. Hu, X. Xiao, W. Sun, C. Wang, P. Feng, Q. D. Shen and Z. Gu, Adv. Mater., 2016, 28, 33133320.

6 Y. Wang, G. Wei, X. Zhang, F. Xu, X. Xiong and S. Zhou, Adv. Mater., 2017, 29, DOI: 10.1002/adma.201605357.

7 J. F. Lovell, T. W. B. Liu, J. Chen and G. Zheng, Chem. Rev., 2010, 110, 2839-2857.

8 X. Zheng, L. Wang, Q. Pei, S. He, S. Liu and Z. Xie, Chem. Mater., 2017, 29, 2374-2381.

9 X. Zheng, Z. Li, L. Chen, Z. Xie and X. Jing, Chem.-Asian J., 2016, 11, 1780-1784.

10 D. Zhang, A. Zheng, J. Li, M. Wu, Z. Cai, L. Wu, Z. Wei, H. Yang, X. Liu and J. Liu, Adv. Sci., 2017, 4, DOI: 10.1002/ advs.201600460.

11 B. Liu, C. Li, G. Chen, B. Liu, X. Deng, Y. Wei, J. Xia, B. Xing, P. a. Ma and J. Lin, Adv. Sci., 2017, 4, DOI: 10.1002/ advs.201600540.

12 S. Niu, W. J. Jiang, T. Tang, Y. Zhang, J. H. Li and J. S. Hu, Adv. Sci., 2017, 4, DOI: 10.1002/advs.201700084.

13 K. Zhu, G. Liu, J. Hu and S. Liu, Biomacromolecules, 2017, 18, 2571-2582.

14 Q. Mou, Y. Ma, X. Zhu and D. Yan, J. Controlled Release, 2016, 230, 34-44.

15 J. Zou, Z. Yin, K. Ding, Q. Tang, J. Li, W. Si, J. Shao, Q. Zhang, W. Huang and X. Dong, ACS Appl. Mater. Interfaces, 2017, 9, 32475-32481.

16 J. Zhang, L. Wang and Z. Xie, ACS Biomater. Sci. Eng., 2017, DOI: 10.1021/acsbiomaterials.7b00470.

17 F. Zhang, G. Zhu, O. Jacobson, Y. Liu, K. Chen, G. Yu, Q. Ni, J. Fan, Z. Yang, F. Xu, X. Fu, Z. Wang, Y. Ma, G. Niu, X. Zhao and X. Chen, ACS Nano, 2017, 11, 8838-8848.

18 U. Bhattacharyya, B. Kumar, A. Garai, A. Bhattacharyya, A. Kumar, S. Banerjee, P. Kondaiah and A. R. Chakravarty, Inorg. Chem., 2017, 56, 12457-12468.

19 Z. Wang and J. Zhao, Org. Lett., 2017, 19, 4492-4495.

20 Y. Liu, Z. Li, L. Chen and Z. Xie, Dyes Pigm., 2017, 141, 5-12.

21 Q. Pei, X. Hu, S. Liu, Y. Li, Z. Xie and X. Jing, J. Controlled Release, 2017, 254, 23-33.

22 S. Y. Qin, A. Q. Zhang, S.-X. Cheng, L. Rong and X. Z. Zhang, Biomaterials, 2017, 112, 234-247.

23 X. Han, J. Chen, M. Jiang, N. Zhang, K. Na, C. Luo, R. Zhang, M. Sun, G. Lin, R. Zhang, Y. Ma, D. Liu and Y. Wang, ACS Appl. Mater. Interfaces, 2016, 8, 33506-33513.

24 W. Zhang, W. Lin, Q. Pei, X. Hu, Z. Xie and X. Jing, Chem. Mater., 2016, 28, 4440-4446.

25 W. Zhang, W. Lin, X. Zheng, S. He and Z. Xie, Chem. Mater., 2017, 29, 1856-1863.

26 A. Loudet and K. Burgess, Chem. Rev., 2007, 107, 4891-4932.

27 Y. Cai, P. Liang, Q. Tang, W. Si, P. Chen, Q. Zhang and X. Dong, ACS Appl. Mater. Interfaces, 2017, 9, 30398-30405.

28 W. Wu, G. Feng, S. Xu and B. Liu, Macromolecules, 2016, 49, 5017-5025.
29 S. Wang, X. Zhao, J. Qian and S. He, RSC Adv., 2016, 6, 4061540625.

30 X. Gu, R. T. K. Kwok, J. W. Y. Lam and B. Z. Tang, Biomaterials, 2017, 146, 115-135.

31 D. Zhang, A. Zheng, J. Li, M. Wu, L. Wu, Z. Wei, N. Liao, X. Zhang, Z. Cai, H. Yang, G. Liu, X. Liu and J. Liu, Theranostics, 2017, 7, 164-179.

32 Y. Zhou, H. Sun, F. Wang, J. Ren and X. Qu, Chem. Commun., 2017, 53, 10588-10591.

33 Y. Shen, Y. Sun, R. Yan, E. Chen, H. Wang, D. Ye, J.-J. Xu and H. Y. Chen, Biomaterials, 2017, 148, 31-40.

34 Y. Liu, X. Hu, L. Wang, X. Liu, T. Bing, W. Tan and D. Shangguan, Dyes Pigm., 2017, 145, 168-173.

35 J. Fu, Y. Zhu and Y. Zhao, J. Mater. Chem. B, 2014, 2, 35383548.

36 S. Shrestha, Z. Mao, Y. Fedutik and C. Gao, J. Mater. Chem. B, 2016, 4, 6955-6966.

37 L. Liu, J. Wang, X. Tan, X. Pang, Q. You, Q. Sun, F. Tan and N. Li, J. Mater. Chem. B, 2017, 5, 2286-2296.

38 S. Liu, D. Lu, X. Wang, D. Ding, D. Kong, Z. Wang and Y. Zhao, J. Mater. Chem. B, 2017, 5, 4918-4925.

39 G. Gao, Y.-W. Jiang, J. Yang and F.-G. Wu, Nanoscale, 2017, 9, 18368-18378.

40 J. Guan, J. Sun, F. Sun, B. Lou, D. Zhang, V. Mashayekhi, N. Sadeghi, G. Storm, E. Mastrobattista and Z. He, Nanoscale, 2017, 9, 9190-9201.

41 Y. Li and C. Zhu, RSC Adv., 2016, 6, 28110-28120.

42 J. H. Lee, N. Mandakhbayar, A. El-Fiqi and H.-W. Kim, Acta Biomater., 2017, 60, 93-108.

43 T. Yin, J. Liu, Z. Zhao, Y. Zhao, L. Dong, M. Yang, J. Zhou and M. Huo, Adv. Funct. Mater., 2017, 27, 1604620.

44 X. Xu, Y. Li, Q. Liang, Z. Song, F. Li, H. He, J. Wang, L. Zhu, Z. Lin and L. Yin, ACS Appl. Mater. Interfaces, 2017, 10, 256266.

45 V. S. Gaware, M. Håkerud, A. Juzeniene, A. Høgset, K. Berg and M. Másson, Biomacromolecules, 2017, 18, 1108-1126.

46 J. Tian, L. Xu, Y. Xue, X. Jiang and W. Zhang, Biomacromolecules, 2017, 18, 3992-4001.

47 K. Han, Z. Ma and H. Han, J. Mater. Chem. B, 2018, 6, 25-38. 48 O. J. Norum, A. S. V. Fremstedal, A. Weyergang, J. Golab and K. Berg, J. Controlled Release, 2017, 268, 120-127.

49 D. Yao, S. Li, X. Zhu, J. Wu and H. Tian, Chem. Commun., 2017, 53, 1233-1236.

50 J. Zhang, D. Chang, Y. Yang, X. Zhang, W. Tao, L. Jiang, X. Liang, H. Tsai, L. Huang and L. Mei, Nanoscale, 2017, 9, 3269-3282.

51 J. Jiang, X. Tian, C. Xu, S. Wang, Y. Feng, M. Chen, H. Yu, M. Zhu and X. Meng, Chem. Commun., 2017, 53, 3645-3648.

52 H. Xiao, C. Wu, P. Li, W. Gao, W. Zhang, W. Zhang, L. Tong and B. Tang, Chem. Sci., 2017, 8, 7025-7030.

53 K. Ma, D. Fu, D. Yu, C. Cui, L. Wang, Z. Guo and C. Mao, Biomaterials, 2017, 121, 55-63.

$54 \mathrm{~J}$. Fu and Y. Zhu, J. Mater. Chem. B, 2017, 5, 996-1004.

55 M. Zhou, X. Du, W. Li, X. Li, H. Huang, Q. Liao, B. Shi, X. Zhang and M. Zhang, J. Mater. Chem. B, 2017, 5, 44554469. 
56 X. Zhang, H. Tan, Y. Yan, Y. Hang, F. Yu, X. Qu and J. Hua, J. Mater. Chem. B, 2017, 5, 2172-2180.

57 S. Daum, M. S. V. Reshetnikov, M. Sisa, T. Dumych, M. D. Lootsik, R. Bilyy, E. Bila, C. Janko, C. Alexiou, M. Herrmann, L. Sellner and A. Mokhir, Angew. Chem., Int. Ed., 2017, 56, 15545-15549.
58 Y. C. Lin, J. F. Lin, T. F. Tsai, H. E. Chen, K. Y. Chou, S. C. Yang, Y. M. Tang and T. I. S. Hwang, Sci. Rep., 2017, 7, 14103.

59 L. Liu, N. Zhang, Y. Dou, G. Mao, C. Bi, W. Pang, X. Liu, D. Song and H. Deng, Sci. Rep., 2017, 7, 41862.

60 Y. Sun, H. Hu, N. Zhao, T. Xia, B. Yu, C. Shen and F. J. Xu, Biomaterials, 2017, 117, 77-91. 